\title{
[gw22-e0552] IN VIVO IMAGING OF ATHEROSCLEROTIC VULNERABLE PLAQUES OF RABBIT MODEL WITH PET/CT
}

Zhao Xin, Zhao Quanming Anzhen Hospital, Beijing, China

\subsection{6/heartjnl-2011-300867.706}

Background and Purpose The rupture of atherosclerotic plaques and the subsequent formation of thrombi are the main factors responsible for myocardial and cerebral infarctions. Thus, the detection of vulnerable plaques in atherosclerotic lesions is a desirable goal, and attempts to image these plaques with 18F-FDG have been made. The aim of our study was to investigate the characteristic of PET/CT imaging for atheromatous plaque evolution as well as the feasibility and accuracy of detecting vulnerable plague using mixed imaging techniques.

Method The 20 male New Zealand white rabbits were divided into three groups: control group $(n=2)$, high cholesterol diet group $(n=9)$ and drug treatment group $(n=9)$. The control group was given normal diet for 18 weeks, in the other two groups, atherosclerosis was induced with a high cholesterol diet fed 2 weeks prior to and 6 weeks after balloon injury of the aorta. After that, the cholesterol diet group fed with normal diet for 6 weeks, followed by 4 weeks of high cholesterol diet. The drug treatment group was given atorvastatin $(1 \mathrm{mg} / \mathrm{day} / \mathrm{kg})$ with normal diet for 10 weeks. 16 weeks after balloon injury, drug-triggering for thrombus was induced by injection of viper venom and histamine. CTA/PET imaging was performed by injection of 18F-FDG at the 18th week before and after the drug-trigger test (control group only before the test), the mean standardised uptake values (SUVmean) and maximal standardised uptake values (SUVmax) were measured over the thoracic and abdominal aortas. After euthanasia, radiational data of arterial segments and histopathological information of arteries were measured.

Results Before the drug-trigger test, maximal standardised uptake value at the control group was $0.473 \pm 0.100$ in control group, $0.658 \pm 0.177$ in drug treatment group, and $0.822 \pm 0.216$ in high cholesterol diet group. After the test, three of four rabbits in high cholesterol diet group were triggered to develop thrombosis, while one of three rabbits in drug treatment group was triggered to develop thrombosis. Thrombotic arteries and non-thrombotic arteries of the high cholesterol diet group compared: SUVmax $(1.390 \pm 0.328$ vs $1.075 \pm 0.159, \mathrm{p}=0.000)$, and the number of macrophage $(57.588 \pm 16.025$ vs $42.795 \pm 13.446$, $\mathrm{p}=0.030$ ), all have statistically significant differences. The number of macrophage was correlated significantly with target-background ratio $(\mathrm{r}=0.449, \mathrm{p}=0.002, \mathrm{n}=42)$, and it has positive correlation between cap/core ratio and target-background ratio $(r=-0.331, p=0.028, n=42)$. The correlation of number of macrophage with 18F-FDG SUVmax was statistically significant $(r=0.399, p=0.007, n=42)$. 
Conclusion The data show that 18F-FDP PET/CT can more comprehensively reflect the characteristics of atherosclerotic lesions at different stages. It has the possibility to become a new method to assess the stability of atherosclerotic plaque and predict thrombotic events for patients, and to some extent, 18F-FDG PET should be useful for evaluating the therapeutic effect of drugs clinically. 